\title{
Surgical Audit
}

\author{
Ogendo S.W.O., MMed, FCS (ECSA), University of Nairobi
}

A good way to describe the first surgical audits is that they were 'polite, restrained discussions'. This was the situation before the development of quality assurance in the business world. As this slowly infiltrated into the medical profession the discussions changed to more criteria based surgical audits.

Surgical audit forms an indispensible part of the practice of surgery today. Audits provide a measure of current performance, reduce clinical risk to the patients and personnel and ultimately aim to improve patient care. The audit process also evaluates the institutional practices and by extension national standards of health care delivery.

A lot of the developments in medicine related to modern audit originated from work in cardiothoracic surgery. With the high cardiac patient turn over in the United States and United Kingdom, a system of audit developed to monitor unit progress.

Why conduct audits? The six main reasons for conducting audits are; as a result of local clinical interests, clinical incident reporting, to comply with regional or national initiatives, to inform patients about surgical results, drive continuous quality improvement and for health care regulation

Surgical units that have regular auditing processes have a clearer idea of the situational analysis of the workings of the unit. This ultimately translates into better management, better planning and review of mistakes. Also with proper functioning units staff moral is elevated. A good unit should have regular reports and learn from clinical incidences and near misses. Key performance indicators like major complications, readmissions, reoperations, transfers, incident reports, complaints and mortalities must also be included.

Surgical audits bear very similar relationship to opera- tional research. There is a critical need for the results to be representative and accurate. Data has to capture all aspects of the audit process and is representative and all aspects of care delivery.

In the POSSUM paper in this issue, the authors audited the results of a very common operative procedure in their institution, the laparatomy. This is probably the most common major operative procedure performed in this country as well as others. Using a comparative audit tool, the results are close to other internationally accepted figures.

The study provides us with useful audit for the facility that can be inserted into the traditional audit model. This can then be reevaluated and even better laparatomy results thereafter produced. Better results will benefit patients, staff, management and finally the nation as a whole. Having read the article I find it a very interesting article, but what next for the practice of laparatomy in the country? This drives home an issue that occurs again and again, good papers are written but no follow-up audit plan is instituted.

As a medical student I clearly recall well attended weekly morbidity and mortality conferences. What happened that we no longer hold such important sessions regularly? These would be ideal fora for dissemination of this important type of information. It was also a useful forum for formulation of institutional (and indeed national) protocols. Protocols collectively owned by the profession provide a unified approach at national level to patient care.

In some countries, surgical audit is facilitated by the local Surgical Colleges or Societies. This process becomes a requirement for annual registration as a component of the Continuous Professional Development programme. Should this be the way for us? 
Probably this avenue needs to be given some consideration as we have no idea of the standard of surgical care being offered in this country. Secondly, morbidity, and mortality figures are not a public issue or even available to surgeons, or the Medical Practitioners and Dentists Board on demand for discussion. Thirdly the collection of audit data needs to be a culture within the surgical profession. At the moment this is not the case.

The local professional medical societies need to take a lead role in improving medical care within this country through auditing. A challenge is to them to formulate an action plan to improve medical audit in the country. Who should collect this data, how should the data be collected, when it is collected and reviewed should be the focus of this action plan.

In Australia and New Zealand this has been formulated and surgeons are required to provide information on their workload, morbidity and mortality and even patient satisfaction figures. This can then be used at na- tional level to audit the country process.

Could it be that the time has come for our surgeons to be required to go the same way? I think this would be a suitable time to go along this road for the sake of the standard of surgical care in this country.

\section{References}

Sinclair A. and Bridgewater B. How to set up a prospective surgical audit. http://www.blackwellpublishing. com/content/BPL_Images/Content_store/Sample_chapter/9781405162685/9781405162685_4_001.pdf. Accessed on $14 / 02 / 10$.

David A. K. Watters, Anthony J. Green, Andrew van Rij. Guidelines for surgical audit in Australia and New Zealand. ANZ J. Surg. 2006; 76: 78-83

Wright JE. The history of surgical audit. J Qual Clin Pract. 1995 Jun; 15(2):81-8.

Christian T Brown. Mark Emberton. Surgical audit and the evaluation of surgery. Surgery. 2006;24(8):276 - 278. 